bei Te, ist die amorphe Phase leicht zu verwirklichen, ebenso auch bei den Gittern mit Schichtbildung wie $\mathrm{P}, \mathrm{As}, \mathrm{Sb}$, weniger bei $\mathrm{Bi}$. Beide Gittertypen sind durch die Gitterdistanz ausgezeichnet, d. h. durch eine schwache Bindung von Kette zu Kette bzw. von Schicht zu Schicht. Diese Bindung geht offenbar im amorphen $\mathrm{Se}, \mathrm{As}$ und $\mathrm{Sb}$ verloren. Hier wurden, wie erwartet, Ketten und auch Schichten beobachtet. Bei den übrigen Elementen liegt im Gitter eine gleichartige Atomanordnung in allen drei Raumrichtungen vor, so daß hier die Chance für die Existenz der amorphen Phase vielleicht weniger groß ist, es sei denn, daß im Gitter wie z. B. bei Si und Ge ganze
Atomgruppen (reguläre Tetraeder) als Baueinheiten auftreten. So ist auch bei Si und Ge der amorphe Zustand zu erreichen; hier liegt nach R i chter und Breitling ${ }^{31}$ ebenfalls eine regellose räumliche Anordnung miteinander verknüpfter Ketten vor.

Herrn Prof. Dr. R. G lo ck e r danken wir herzlich für seine fördernden Ratschläge und sein stetes Interesse an dieser Arbeit, ebenso sei auch der Notgemeinschaft der Deutschen Wissenschaft für die Bereitstellung apparativer Hilfsmittel gedankt.

31 H. Richter u. G. B reitling, „Struktur des amorphen Germanium und Silicium." Die Arbeit erscheint in Kürze.

\title{
Die Randschicht eines Germanium-Flächengleichrichters im Flußbereich
}

\author{
Vorläufige Mitteilung \\ Von W. Bösenberg und E. Fues \\ Aus dem Institut für theoretische und angewandte Physik der Technischen Hochschule Stuttgart \\ (Z. Naturforschg. 6 a, 741-744 [1951]; eingegangen am 25. Juli 1951)

\section{Erich Regener zum 70. Geburtstag}

\begin{abstract}
Einige Rechnungen, die über die Feld- und Konzentrationsverteilung der Elektronen und
\end{abstract} \\ Löcher in der S c h o t t k y schen Randschicht eines Metall/Germanium-Kontaktes durchgeführt \\ wurden, lassen den Schwemmeffekt in der Umgebung der Emittorelektrode eines Transistors \\ durch die S chottky sche Theorie verstehen. Bei großen Flußströmen ergibt sich im Halb- \\ leiter eine stark erhöhte Elektronen- und Löcherkonzentration.
}

$I^{n}$ $\mathrm{m}$ Anschluß an eine Arbeit von van R o os bro e ck ${ }^{1}$, der die Feld- und Konzentrationsverteilung der Elektronen und Löcher im halbleitenden Germanium nach Löcherinjektion unter Berücksichtigung der Rekombination, aber unter Vernachlässigung der Raumladung berechnet hat, wurden diese Verteilungen für einen Metall/Germanium-Kontakt mit Inversionsschicht im Bereich der raumladungsbehafteten S chottky schen Randschicht unter Vernachlässigung der Rekombination berechnet. Die Lösungen der nicht-linearen Grundgleichungen [vgl. S chottky ${ }^{2}$, Gln. $(5,10)$ und 5,11$\left.)^{*}\right]$ sind nicht geschlossen erhältlich. Genaue Lösungen wurden mit einem numerischen Iterations. verfahren nach v on $\mathrm{S}$ a n d e ${ }^{3}$ für den Strom Null und für einen großen Flußstrom bei Germanium mit

1 W. vản Roosbroeck, Bell System Techn. J. 29, 560 [1950].

2 W. S ch ot tk y u. E. S pe nke, Wiss. Veröff. Siemens-Konzern 18, 225 [1939].

3 H. von S a nden, Praxis der Differentialgleichungen, W. de Gruyter, Berlin 1945.

4 W. S c h o t tky, Z. Physik 113, 367 [1939]. einer Störstellendichte von $10^{15} \mathrm{~cm}^{-3}$ berechnet. Nach $\mathrm{S} \mathrm{ch} \mathrm{ot} \mathrm{t} \mathrm{k} \mathrm{y}{ }^{4}$ sollte sich das thermische Gleichgewicht der Metallelektronen auch bei großen Flußströmen auf den Halbleiter auswirken und Anlaß zu stromunabhängigen Randwerten der Elektronen- und Löcherkonzentrationen - dies wurde als zulässige Randbedingung angesehen - geben. Die zugehörige elektrische Randfeldstärke wurde durch Probieren so bestimmt, daß sich von einem endlichen Wert der Ortskoordinate ab, nämlich am Ende der Randschicht, Raumladungsfreiheit ergibt, was weiterhin - immer unter Vernachlässigung der Rekombination - zu konstanten Werten der Elektronen- und Löcherdichte und zu einem konstanten Wert der Bahnfeldstärke führen würde. Die weitere Lösung sollte aber nach $\mathrm{v}$ a n $\mathrm{Roosbroeck^{1 }}$ fortgesetzt werden.

* Die Gl. $(5,10)$ von 2 ist einmal für die Elektronenkonzentration $p$ und einmal für die Löcherkonzentration $q$ anzuschreiben. In Gl. $(5,11)$ ist statt $f(p)$ die stromabhängige Differenz $q-p$ gegen die Werte für den Strom Null im raumladungsfreien Halbleiterinneren einzusetzen. 
Die numerischen Rechnungen sind sehr mühselig, und es wurde daher nach einem analytischen Nähe-
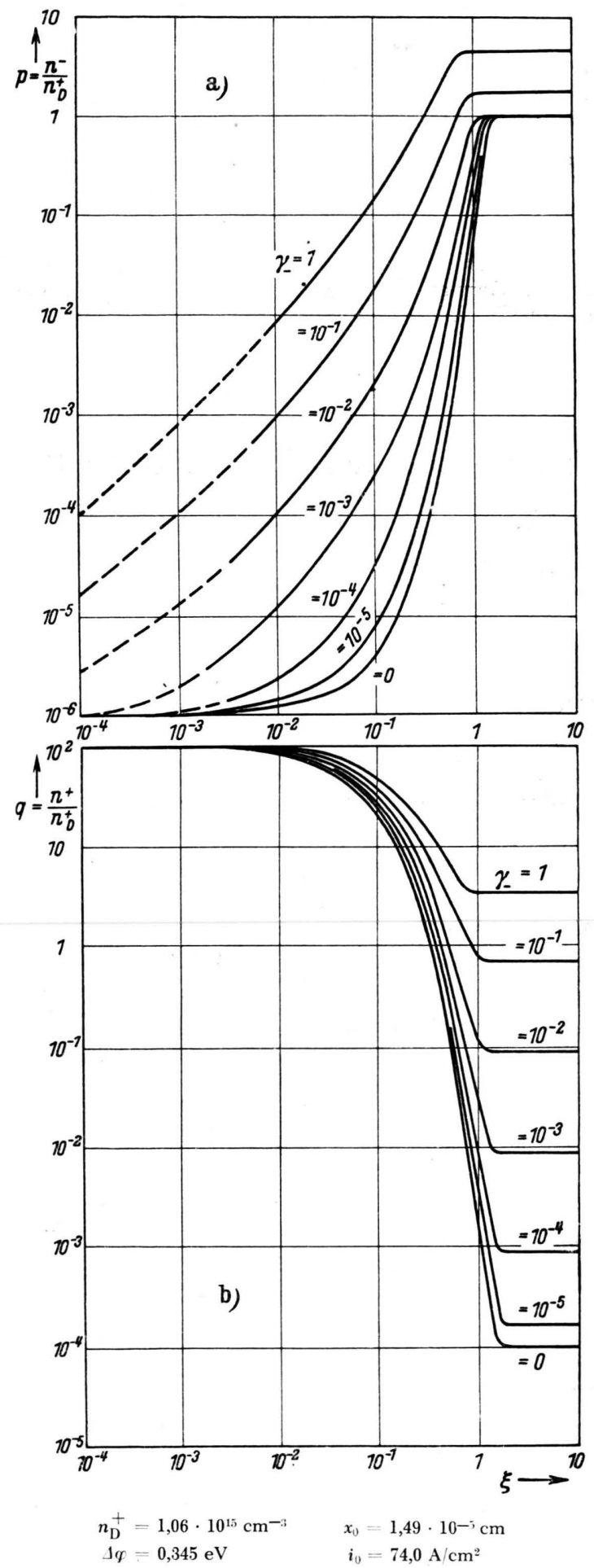

Abb. $1 \mathrm{a}$ und $1 \mathrm{~b}$. rungsverfahren gesucht. Nach dem Vorbild der Schottkyschen „vereinfachten Theorie“ ${ }^{5,6,7}$ wurde deshalb zweitens im Bereich der Randschicht konstante Raumladung und daher eine lineare Feldverteilung angenommen. Leider ist die Raumladung bei bipolarer Leitfähigkeit nicht nur von den thermischen und materiellen Eigenschaften des Halbleiters abhängig, sondern ist infolge der hohen Löcherdichte der Inversionsschicht und durch deren Schwemmverhalten selbst stark orts- und stromabhängig. Die „vereinfachte Theorie" besteht deshalb hier nicht mit gleichem Recht wie bei Schottky. Es ergeben sich aber mathematische Schwierigkeiten, wenn man z. B. das Feld durch einen Polynomausdruck darstellt, da die hierbei auftretenden Integrale nicht mehr genügend tabuliert sind. Außerdem ist die Art der Mittelung der strom- und ortsabhängigen Raumladung sehr wesentlich (und einstweilen nicht ganz willkürfrei) für den Verlauf der Konzentrationsverteilungen. Während sich für den Strom Null noch ein allgemeines Integral für die Randfeldstärke finden läßt, ist das bei den Flußströmen, die hauptsächlich interessieren, nicht mehr der Fall. Aus der eben erwähnten Randfeldstärke wurde nun eine Dicke der Randschicht bestimmt, sie ergibt sich aber auf diese Art um etwa

5 W. S c h o t t k y, Z. Physik 118, 539 [1941].

6 E. S p enk e, Z. Physik 126, 67 [1949].

7 Man erhält allgemein eine Formel vom Typ $(1,28)$ vcn 6 für $p$ und bei vertauschtem Vorzeichen der Exponenten der Exponentialfunktionen auch für $q$.

Legende zu nebenstehender Abbildung :

Abb. $1 \mathrm{a}$ und $1 \mathrm{~b}$. Die relative Elektronenkonzentration $p=n^{-} / n_{\mathrm{D}}^{+}$(Abb. 1 a) und die relative Löcherkonzentration $q=n^{+} / n_{\mathrm{D}}^{+}$(Abb. $1 \mathrm{~b}$ ) nach der ,vereinfachten Theorie ${ }^{\text {“ }}$ in Abhängigkeit von der relativen Ortskoordinate $\xi=x / x_{0}$ $\left(x_{0}=\sqrt{\frac{\varepsilon \cdot \mathfrak{B}}{4 \pi e n_{\mathrm{D}}^{+}}}\right.$auf reine Elektronenleitung bezogen!)in doppelt logarithmischem Maßstab. Als Parameter dient die relative Elektronenstromdichte $\gamma_{-}=i^{-} / i_{0}\left(i_{0}=e n_{\mathrm{D}}^{+} b^{-} \cdot 2 \mathfrak{S} / x_{0}\right.$ mit $\mathfrak{B}=k T / e, \varepsilon=\mathrm{DK}$ und $b^{-}$als Elektronenbeweglichkeit), die mit der relativen Gesamtstromdichte $\gamma=i / i_{0}$ nach der folgenden Tabelle zusammenhängt:

\begin{tabular}{cccccccc}
$\gamma_{-}=0$ & $10^{-6}$ & $10^{-5}$ & $10^{-4}$ & $10^{-3}$ & $10^{-2}$ & $10^{-1}$ & 1 \\
\hline$\gamma^{\prime} / \gamma_{-}=1,00$ & 1,00 & 1,00 & 1,00 & 1,01 & 1,06 & 1,28 & $1,52^{*}$
\end{tabular}

* Es ist $\gamma=\gamma_{-}\left(1+\frac{b^{+} q_{\mathrm{HL}}^{\gamma}}{b^{-} p_{\mathrm{HL}}^{\gamma}}\right)$, also ist asymptotisch $\gamma \sim \gamma_{-}\left(1+\frac{b^{+}}{b^{-}}\right) ;$mit $b^{-} / b^{+}=1,5$ ergibt sich numerisch $\gamma \sim 1,67 \gamma_{-}$. 
den Faktor 5 zu dünn. Eine wesentlich bessere Darstellung würde man erhalten, wenn man eine $\mathrm{Ab}$ schätzung für die mit der Stromstärke wechselnde Dicke der Randschicht hätte und die Mittelung der Raumladung über diese Randschicht durchführen würde. Es würde sich dann über einen sehr schmalen Bereich am Rand ein falscher Wert der Randfeldstärke um etwa den Faktor 2 bis 5 ergeben, doch wäre das wohl bei der gewonnenen Rechenerleichterung tragbar. Bisher ist es indessen noch nicht gelungen, einen willkürfreien Ausdruck für die Randschichtdicke bei bipolarer Leitfähigkeit in Abhängigkeit vom Strom

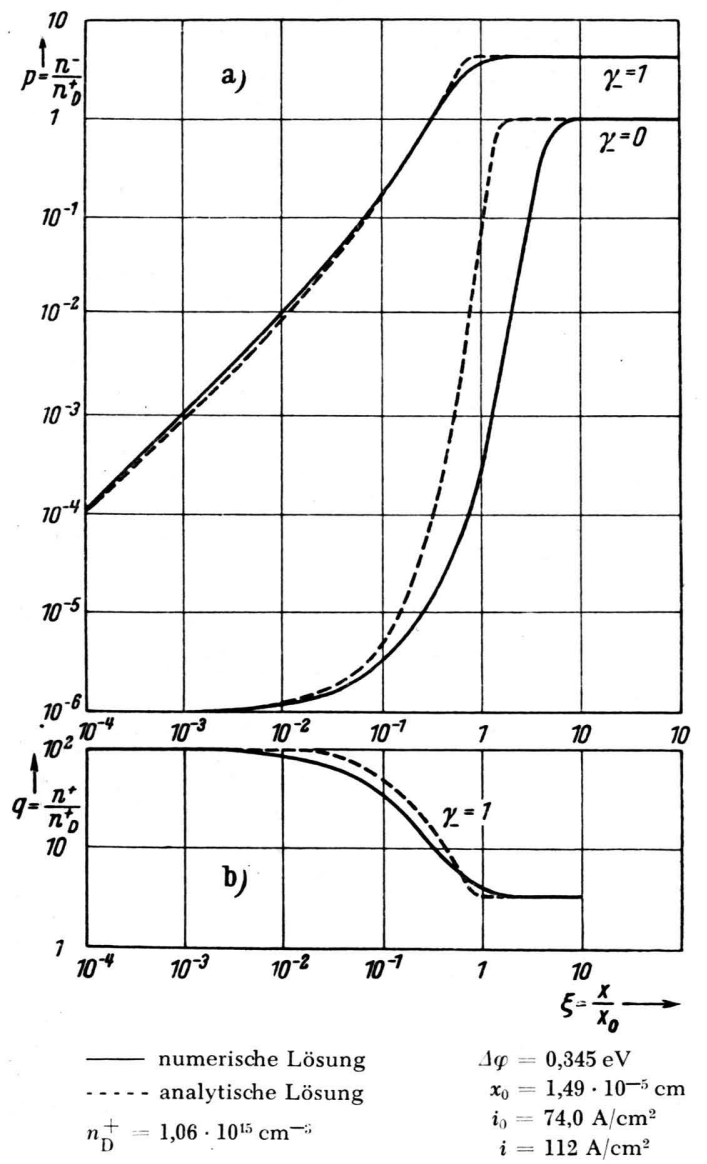

Abb. 2 a und 2 b. Vergleich der analytisch nach der ,vereinfachten Theorie“ gewonnenen relativen Elektronendichte $p=n^{-} / n_{\mathrm{D}}^{+}$(Abb. $2 \mathrm{a}$ ) und Löcherdichte $q=n^{+} / n_{\mathrm{D}}^{+}$ (Abb. 2 b) mit der durch numerische Integration gewonnenen. Abszisse ist die relative Ortskoordinate $\xi=x / x_{0}$, als Parameter dient die relative Elektronenstromdichte $\gamma_{-}=i^{-} / i_{0}$. Da der Maßstab doppelt logarithmisch ist, erübrigt sich beim thermischen Gleichgewicht $p q=K(T)$ wegen $\log q=\log K(T)-\log p$ das Auftragen der Kurve $q$ für den Strom Null. zu finden, daher soll diese Mitteilung als vorläufig betrachtet werden. Wir hoffen, die analytisch berechneten Kurven später zu verbessern.
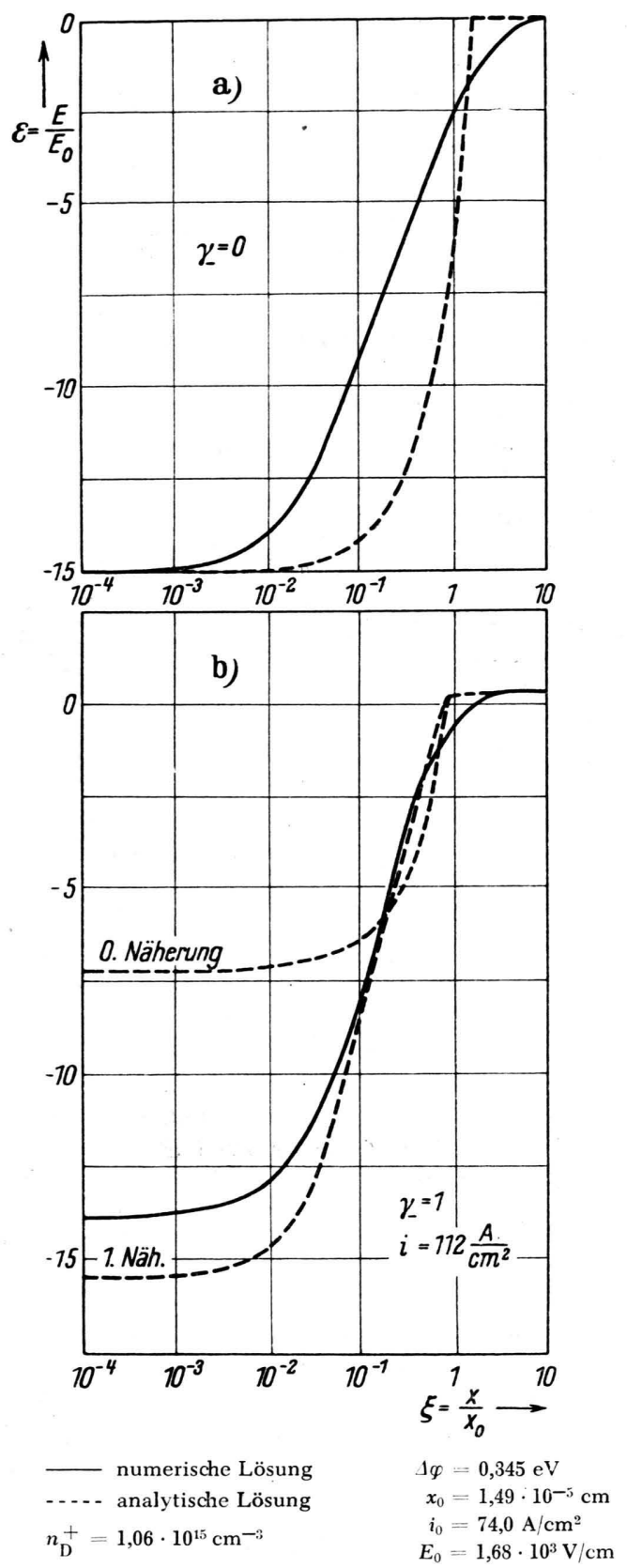

Abb. 3 a und 3 b. Vergleich der bei der ,vereinfachten Theorie“ verwendeten relativen Feldstärke $\varepsilon=E / E_{0}$ $\left(E_{0}=\mathfrak{B} / x_{0}\right)$ in nullter Näherung linear von $\xi$ abhängig!) mit der durch numerische Integration gewonnenen. Als Abszisse dient im doppelt logarithmischen Maßstab die relative Ortskoordinate $\xi=x / x_{0}$. Parameter ist die relative Elektronenstromdichte $\gamma_{-}=i-/ i_{0}$. 
Aber schon im jetzigen Zustand lassen sie und besser noch die numerisch berechneten Lösungen den typischen Schwemmeffekt erkennen. Die Löcher werden bei starken Flußströmen in hohem Maß ins Halbleiterinnere hineingerissen, erzeugen dort auch eine erhöhte Elektronenkonzentration und führen zu recht erheblichen Abweichungen vom thermischen Gleichgewichtszustand.
Die Daten des durchgerechneten Beispiels

$n$-Germanium einer Störstellendichte $n_{\mathrm{D}}^{+}$von rund $10^{15} \mathrm{~cm}^{-3}$, alle Donatoren bei $T=300^{\circ} \mathrm{K}$ ionisiert, Austrittsarbeit Metall/Halbleiter $0,345 \mathrm{eV}$ (es ergeben sich also eine Elektronen-Randkonzentration von rd. $10^{9} \mathrm{~cm}^{-3}$ und eine Löcher-Randkonzentration von $\mathrm{rd}$. $\left.10^{17} \mathrm{~cm}^{-3}\right)$.

\section{BESPRECHUNGEN}

Vorlesungen über höhere Mathematik. Von A d a l ber t D u s c h e k. 1. Band. Springer-Verlag, Wien 1949. 395 S. mit 167 Abb.; Preis DM 26.-.

Vorlesungen über höhere Mathematik. Von A d a l b e r t D u s chek. 2. Band. Springer-Verlag, Wien 1950. 386 S. mit 125 Abb.; Preis DM 26.-.

Der 1. Band behandelt, von Zahlenfolgen und Funktionsbegriff ausgehend, erst das Bestimmte Integral, dann die Ableitung, Elementare Transzendenten, legt dann die Differential- und Integralrechnung eine Schicht tiefer, bis zur Taylorschen Formel und Reihe und zur Numerischen Integration und schließt mit einer zusammenfassenden Darstellung über Polynome, Rationale Funktionen und Algebraische Gleichungen; dabei werden Verfahren für das praktische Rechnen besonders berücksichtigt. Zum Unterschied von allen geläufigen Darstellungen hat der Verf. die heute für das naturwissenschaftliche Denken wesentliche Wahrscheinlichkeitsrechnung von den ersten Abschnitten des Werkes an mitbehandelt und auf jeder Stufe den erreichten Kenntnissen entsprechend weitergeführt. Natürlich beginnt er mit einfachen Definitionen der Wahrscheinlichkeit und verzichtet auf Erörterung von Grundlagenfragen.

Der 2. Band umfaßt: Unendliche Reihen, Funktionen mehrerer Veränderlichen in bezug auf Differentiation und Integration, Fundamentalsätze der Wahrscheinlichkeitsrechnung, Fehlertheorie und Ausgleichsrechnung, Lineare Algebra mit besonderer Blickrichtung auf Vektoren und Tensoren 2. Stufe sowie etwas Tensoranalysis. Die Auswahl ist wohl abgewogen und als sehr gelungen anzuerkennen. Eine große Zahl von Beispielen und Übungsaufgaben (mit Lösungen am Schluß des Bandes) belebt die Darstellung. Auch die Ausstattung ist vorzüglich. Für eine sicher zu erwartende Neuauflage bleibt nur zu wünschen, daß die Diktion gestrafft werde, die an manchen Stellen für den Lernenden verschiedene Deutungen offen läßt (eine richtige, und einen Holzweg), und daß auch in einer Anzahl von Beweisen Vollständigkeit herbeigeführt oder der Verzicht auf sie ausdrücklich festgestellt werde; wenn es der Verf. vorzieht, nur einen - meist ausreichen- den - Sonderfall zu beweisen, clie Aussage des Satzes aber auch allgemeiner zu formulieren, so ist dann nichts mehr einzuwenden. Man sollte aber die jungen Leser nicht über die Tragweite von Beweisen im unklaren lassen. - Diese Anregungen wollen wir als positive Kritik zum Besten der Zukunft des Werkes verstanden wissen.

Eg on Ullrich, Gießen.

Die veränderlichen Sterne der nördlichen Milchstraße. Teil VI. Von C. H off $\mathrm{m}$ e is te r, E. R o h lf $\mathrm{s}$ und P. A hnert. Veröffentlichungen der Sternwarte in Sonneberg, Band 1, Nummer 5. Akademie-Verlag GmbH., Berlin 1951. 498 S. mit 54 Abb.; Preis DM 13.-

Die vorliegende Publikation stellt die 6. Fortsetzung der Veröffentlichungen des Sonneberger Plans zur Erforschung der veränderlichen Sterne ausgewählter Milchstraßenfelder dar und schließt sich den früheren eng an. Behandelt werden die Felder um $\varkappa$ Lyrae (92 Sterne), $\varrho$ Cygni (127 Sterne) und 130 Tauri (39 Sterne). Die Helligkeiten wurden auf Platten des 400-mm-Astrographen und zweier kleinerer Triplet-Kameras, gelegentlich auch visuell am Fernrohr, bestimmt. Bis auf 21 Objekte konnte für alle Veränderlichen die Art des Lichtwechsels geklärt werden. Die statistische Auswertung des Materials wurde wie bisher für eine abschließende Diskussion zurückgestellt.

\section{G. R. M i c z a i k a, Heidelberg.}

Die Veröffentlichung dieser Festschrift im vorliegenden Umfang erfolgt mit freundlicher Unterstützung durch eine Reihe von Persönlichkeiten und Firmen, welche damit dem Jubilar ihre Verbundenheit zum Ausdruck bringen.

Der Verlag und der Initiator danken an dieser Stelle herzlichst für diese wertvolle Hilfe. 